\title{
Self-reported anosmia and dysgeusia as key symptoms of coronavirus disease 2019
}

\author{
Daniel J. Lee (1), MD*; Joel Lockwood, MD*; Paul Das, MD, MSc; ; Ri Wang, MMathף; \\ Eitan Grinspun, PhD ${ }^{* *}$; John M. Lee ${ }^{\circ}, \mathrm{MD}, \mathrm{MSc}^{* \dagger}$
}

\author{
CLINICIAN'S CAPSULE \\ What is known about the topic? \\ Patients with coronavirus disease 2019 (COVID-19) pre- \\ sent with various influenza-like symptoms, making it diffi- \\ cult to distinguish from other viral infections for frontline \\ physicians. \\ What did this study ask? \\ Do COVID-19 positive patients have higher prevalence of \\ self-reported anosmia/hyposmia and dysgeusia/ageusia \\ than COVID-19 negative patients? \\ What did this study find? \\ This study found that anosmia/hyposmia and dysgeusia/ \\ ageusia were respectively 14 and 11 times more likely to \\ be associated with COVID-19 positivity. \\ Why does this study matter to clinicians? \\ Using anosmia/hyposmia and dysgeusia/ageusia as spe- \\ cific criteria may lead to improved clinical diagnosis of \\ COVID-19 positive patients.
}

\section{ABSTRACT}

Objectives: To slow down the transmission of coronavirus disease 2019 (COVID-19), it is important to identify specific symptoms for effective screening. While anosmia/hyposmia and dysgeusia/ageusia have been identified as highly prevalent symptoms, there are wide geographic variations, necessitating the regional evaluation of the prevalence of the symptoms. Methods: A cross-sectional study was performed to evaluate the self-reported symptoms among adults (over 18 years old) who underwent COVID-19 tests at an ambulatory assessment centre. We identified 1,345 patients (102 positive and 1,243 negative) who visited the assessment centre between March 16 and April 15, 2020. We randomly sampled negative patients in a 1:3 ratio. The primary outcome was the prevalence of selfreported anosmia/hyposmia and dysgeusia/ageusia. Logistic regression was performed to evaluate the association between COVID-19 positivity and loss of smell and taste.

Results: Fifty-six of 102 (50\%) positive patients and 72 of 306 (23.5\%) negative patients completed the survey. Anosmia/ hyposmia and dysgeusia/ageusia were more prevalent among COVID-19 positive patients $(41.1 \%$ v. $4.2 \%, p<0.001$ for smell and $46.4 \%$ v. $5.6 \%, p<0.001$ for taste). Anosmia/ hyposmia and dysgeusia/ageusia were independently highly associated with COVID-19 positivity (adjusted odds ratios 14.4 and 11.4 for smell and taste, respectively).

Conclusion: In this Canadian study, smell and taste loss may be key symptoms of COVID-19. This evidence can be helpful in the clinical diagnosis of COVID-19, particularly settings of limited testing capacity.

\section{RÉSUMÉ}

Contexte: Afin de ralentir la transmission de la maladie à coronavirus 2019 (COVID-19) par un dépistage efficace, il est important de reconnaître les symptômes spécifiques de la maladie. Bien que l'anosmie/l'hyposmie et la dysgeusie/l'ageusie soient souvent des symptômes concomitants de la maladie, il existe de grandes variations géographiques qui appellent une évaluation régionale de la prévalence des symptômes.

Méthode: II s'agit d'une étude transversale visant à évaluer la pertinence de symptômes autodéclarés de la maladie chez des adultes (plus de 18 ans) ayant subi un test de dépistage de la COVID-19 dans un centre ambulatoire de prélèvements. Ainsi, 1345 patients (102 cas positifs et 1243 cas négatifs) se sont rendus dans un centre de dépistage entre le 16 mars et le 15 avril 2020. L'équipe a par la suite procédé à un échantillonnage aléatoire de patients ayant obtenu un résultat négatif

From the * Department of Otolaryngology - Head and Neck Surgery, St. Michael's Hospital, Unity Health Toronto, University of Toronto, Toronto, $\mathrm{ON}$; ${ }^{\dagger} \mathrm{Li}$ Ka-Shing Knowledge Institute, St. Michael's Hospital, Unity Health Toronto, Toronto, ON; ${ }^{\ddagger}$ Department of Emergency Medicine, St. Michael's Hospital, Unity Health Toronto, Toronto, ON; ${ }^{5}$ Department of Family and Community Medicine, St. Michael's Hospital, Unity Health Toronto, Toronto, ON; "MAP Centre for Urban Health Solutions, Li Ka-Shing Knowledge Institute, St. Michael's Hospital, Unity Health, Toronto, ON; and the ${ }^{* *}$ Department of Computer Science, University of Toronto, Toronto, ON.

Correspondence to: Dr. John M. Lee, St. Michael's Hospital, 30 Bond Street, 8 Cardinal Carter Wing, Toronto, ON M5B 1W8; Email: John.Lee@ unityhealth.to.

(C) Canadian Association of Emergency Physicians 2020 CJEM 2020;22(5):595-602

DOI $10.1017 /$ cem. 2020.420

This is an Open Access article, distributed under the terms of the Creative Commons Attribution licence (http://creativecommons.org/licenses/by/4.0/), which permits unrestricted re-use, distribution, and reproduction in any medium, provided the original work is properly cited. 
dans un rapport de 1/3. Le critère d'évaluation principal était la prévalence des symptômes autodéclarés d'anosmie/d'hyposmie et de dysgeusie/d'ageusie. L'association entre la positivité des résultats à l'égard de la COVID-19 et la perte de l'odorat et du goût a été examinée à l'aide d'une régression logistique.

Résultats: Dans l'ensemble, 56 patients sur 102 (50\%) déclarés positifs et 72 patients sur $306(23,5 \%)$ déclarés négatifs ont poursuivi I'enquête jusqu'au bout. La prévalence de I'anosmie/l'hyposmie et de la dysgeusie/l'ageusie était plus élevée chez les patients déclarés positifs (odorat : 41,1\% contre [c.] $4,2 \%, p<0,001$; goût : $46,4 \%$ c. $5,6 \%, p<0,001)$ que chez ceux déclarés négatifs. L'anosmie/l'hyposmie et la dysgeusie/l'ageusie se sont révélées des symptômes indépendants, fortement associés à la positivité des résultats à l'égard de la COVID-19 (risque relatif approché rajusté : 14,4 et 11,4 pour I'odorat et le goût, respectivement).

Interprétation: Compte tenu des résultats obtenus dans cette étude réalisée au Canada, la perte de l'odorat et du goût pourrait être considérée comme un symptôme important de la maladie. II s'agirait là d'une manifestation susceptible de faciliter la pose du diagnostic clinique de la COVID-19, tout particulièrement dans les milieux où la capacité de dépistage est restreinte.

Keywords: Anosmia, COVID-19, dysgeusia, epidemiology, population health

\section{INTRODUCTION}

Coronavirus disease 2019 (COVID-19) is an international outbreak of respiratory illness characterized by high transmission rate, particularly in individuals with no or mild symptoms. ${ }^{1,2}$ A key step in minimizing transmission is to screen and isolate those infected with COVID-19 using specific clinical criteria. While several symptoms are used to screen, there is lack of data to suggest specific symptoms of COVID-19. Anosmia/hyposmia and ageusia/dysgeusia have emerged as potential specific symptoms, as reported in recent studies from the United States and Europe. ${ }^{3-5}$ Spinato et al. described $64.4 \%$ of altered smell or taste among Italian adults with mild symptoms and Yan et al. described $68 \%$ and $71 \%$ of anosmia and dysgeusia, respectively, in COVID-19 positive individuals with an odds ratio (OR) of $10 .{ }^{3,4} \mathrm{~A}$ recently published study from Quebec reported 51.5\% of anosmia. ${ }^{6}$ These reports suggest that anosmia/hyposmia and dysgeusia/anosmia have the potential to be used to specifically screen for individuals with mild symptoms. However, there are wide variations in the reported prevalence of anosmia/hyposmia and ageusia/dysgeusia across different regions, potentially suggesting variable geographic presentations of severe acute respiratory syndrome coronavirus 2 (SARS-CoV-2). ${ }^{7}$ Therefore, it is imperative that we further examine this potential association between loss of smell and taste and COVID-19 diagnosis in the Canadian and Ontario context. The objective was to evaluate the prevalence and association of anosmia/hyposmia and dysgeusia/ageusia in patients who underwent COVID-19 testing using self-reported measures.

\section{METHODS}

\section{Study design and patient population}

The approval of this study was granted by the Research Ethics Board at Unity Health Toronto, Toronto, Ontario, through Clinical Trials Ontario (CTO ID: 2142). We designed a cross-sectional survey of adults (over 18 years of age) who had undergone polymerase chain reaction-confirmed COVID-19 testing via nasopharyngeal swab at the COVID-19 Assessment Centre at St. Michael's Hospital, Toronto, Ontario, between March 16 and April 15, 2020. Patients were contacted by phone for an invitation to a secure online survey, which was constructed using Snap Software, fully compliant with the Personal Health Information Protection Act, after being notified of the results of their swab. During this period, we identified 102 unique COVID-19 positive patients and 1,243 unique negative patients. Due to a large number of negative patients, we randomly sampled negative patients in a 1:3 ratio.

\section{Outcome measures and data collection}

Baseline characteristics were collected and included: age, gender, medical comorbidities, and smoking status. We listed chronic rhinosinusitis and history of recent severe upper respiratory tract infection (URTI) or flu as a separate comorbidity, as these may impact baseline sense of smell. COVID-19 diagnosis, symptoms, and hospitalizations were collected. Smell and taste-specific questions included the presence of smell or taste loss around the onset of COVID-19-like symptoms (5 days earlier or 
any time after), as well as the current ability to smell. The type of taste loss was collected (sweet, salty, sour, bitter, and savoury).

\section{Statistical analysis}

Demographic and clinical characteristics were summarized descriptively by reporting the median and interquartile (IQR) range for continuous variables and the frequency and proportion for categorical variables. Differences in characteristics between two comparison groups were compared using the Kruskal-Wallis test for continuous variables and Fisher's exact test for categorical variables. Unadjusted ORs were calculated with univariable logistic regression. Adjusted OR was calculated using multivariable logistic regression analysis with a priori criteria. To prevent over-fitting, we included the loss of smell or taste around the onset of COVID-19-like symptoms and four other covariates with a statistically significant magnitude of association of at least twofold (i.e., $\mathrm{OR}>2.0$ or $\mathrm{OR}<0.5$ ).

\section{RESULTS}

Of 102 positive patients and 306 negative patients who were contacted, $56(50 \%)$ positive patients and $72(23.5 \%)$ negative patients completed the survey between April 15 and May 1, 2020. Baseline information is summarized in Table 1. Positive patients were younger than negative patients (38.0 IQR 31.8-47.2 v. $43.0 \mathrm{IQR}$ 33.5-55.0, $p<0.05)$. Gender, smoking status, and comorbidities relevant to smell function (head trauma, chronic rhinosinusitis, and recent URTI/flu) were wellbalanced. There was a longer time lapse between the diagnosis and the survey among the negative patients compared with the positive patients $(67.6 \%$ for negative v. $30.4 \%$ for positive for more than 4 weeks since the diagnosis).

The overall symptoms are summarized in Table 1. A significantly higher proportion of positive patients reported anosmia $(42.9 \%$ v. $2.8 \%, p<0.001)$, hyposmia $(12.5 \%$ v. $1.4 \%, p<0.05)$ and dysgeusia/ageusia $(57.1 \%$ v. $1.4 \%, p<0.001)$ compared with negative patients. In addition, cough, fever, diarrhea, and headache were more common among positive patients. On the other hand, sore throat was more common among negative patients $(63 \%$ v. $37 \%, p<0.01)$.
Characterization of anosmia/hyposmia and dysgeusia/ ageusia is summarized in Table 2. When patients were inquired about their smell loss around the time of COVID-19-like symptom onset (5 days before or any time after), there was a significantly higher proportion of COVID-19-positive patients compared with the negative patients ( $41.1 \%$ v. $4.2 \%, p<0.001)$. Of 23 positive patients with anosmia/hyposmia, 12 (52.2\%) patients reported that anosmia/hyposmia was one of the early symptoms. There was a significantly higher proportion of positive patients reporting dysgeusia/ ageusia compared with negative patients $(46.4 \% \mathrm{v}$. $5.6 \%, p<0.001)$. Twenty $(35.7 \%)$ positive patients reported concomitant smell and taste loss.

On the univariable analysis, anosmia/hyposmia and dysgeusia/ageusia were highly associated with COVID-19 positivity OR 19.7 (95\% confidence interval [CI] 6.188.7) and OR 13.2 (95\% CI 4.6-48.0), respectively (Table 3). Fever, cough, sore throat, and headache were included in the multivariable analysis with either anosmia/hyposmia or dysgeusia/ageusia. In the multivariable models, smell loss and taste loss demonstrated high adjusted ORs with COVID-19 positivity (OR 14.4 [95\% CI 4.0-70.5] and OR 11.4 [95\% CI 3.6-45.3], respectively). Sore throat was negatively associated with COVID-19 positivity. Other symptoms were statistically not significant.

There were no differences between patients with and without anosmia/hyposmia in terms of age, smoking, relevant comorbidities (chronic rhinosinusitis, recent URTI/ flu, head trauma), and symptoms (rhinorrhea and nasal congestion) (Table 4). There were significantly more COVID-19-positive patients in the group with smell loss than the one without $(88.5 \%$ v. $28.1 \%, p<0.001)$.

Among those with anosmia/hyposmia $(\mathrm{n}=25,1 \mathrm{did}$ not answer), 13 (52\%) patients reported their sense of smell at the time of survey to be normal. Twelve (48\%) patients who had persistent smell loss were within less than 4 weeks from the diagnosis. All eight patients who completed the survey more than 4 weeks after the diagnosis reported a normal sense of smell.

\section{DISCUSSION}

\section{Interpretation of findings}

This study suggests that there is a higher prevalence of self-reported chemosensory impairment in 
Daniel J. Lee et al.

\begin{tabular}{|c|c|c|c|}
\hline & Total $(n=127)$ & Positive $(n=56)$ & Negative $(n=71)$ \\
\hline Age - median $(I \mathrm{QR})^{*}$ & $41.0(32.0-52.5)$ & $38.0(31.8-47.2)$ & $43.0(33.5-55.0)$ \\
\hline \multicolumn{4}{|l|}{ Gender - \% } \\
\hline Male & 38.6 & 41.1 & 36.6 \\
\hline Female & 60.6 & 58.9 & 62.0 \\
\hline Others & 0.8 & 0.0 & 1.4 \\
\hline \multicolumn{4}{|l|}{ Smoking - \% } \\
\hline Never & 67.7 & 71.4 & 64.8 \\
\hline Current & 9.5 & 5.4 & 12.7 \\
\hline Ex-smoker & 21.3 & 19.6 & 22.5 \\
\hline Others (vape) & 1.6 & 3.6 & 0.0 \\
\hline \multicolumn{4}{|l|}{ Comorbidities - \% } \\
\hline Cardiac & 1.6 & 0.0 & 2.8 \\
\hline Neurological & 0.0 & 0.0 & 0.0 \\
\hline Respiratory & 14.2 & 17.9 & 11.3 \\
\hline Diabetes & 7.1 & 7.1 & 7.0 \\
\hline Hypertension & 13.4 & 10.7 & 15.5 \\
\hline Cancer* & 5.5 & 0.0 & 9.9 \\
\hline Head trauma & 5.5 & 3.6 & 7.0 \\
\hline Chronic rhinosinusitis & 3.9 & 3.6 & 4.2 \\
\hline Recent severe URTI or flu & 9.5 & 8.9 & 9.9 \\
\hline None* & 38.6 & 25.0 & 49.3 \\
\hline \multicolumn{4}{|c|}{ Time of survey completion from time of swab - \% } \\
\hline $0-2$ weeks & 14.2 & 30.4 & 1.4 \\
\hline 2-4 weeks & 34.7 & 39.3 & 31.0 \\
\hline$>4$ weeks & 51.2 & 30.4 & 67.6 \\
\hline Hospitalization - n (\%) & 7.0 & 7.3 & 0.0 \\
\hline \multicolumn{4}{|l|}{ Overall symptoms - n (\%) } \\
\hline Sore throat* & 52.0 & 37.5 & 63.4 \\
\hline Cough* & 52.8 & 66.1 & 42.3 \\
\hline Nasal congestion & 39.4 & 41.1 & 38.0 \\
\hline Rhinorrhea & 36.2 & 26.8 & 43.7 \\
\hline Fever* & 35.4 & 46.4 & 26.7 \\
\hline Shortness of breath & 31.5 & 37.5 & 26.8 \\
\hline Abdominal pain & 10.2 & 12.50 & 8.5 \\
\hline Diarrhea* & 26.0 & 35.7 & 18.3 \\
\hline Anosmia* & 20.5 & 42.9 & 2.8 \\
\hline Hyposmia* & 6.3 & 12.5 & 1.4 \\
\hline Dysgeusia/ageusia* & 26.0 & 57.1 & 1.4 \\
\hline Fatigue & 11.8 & 7.1 & 15.5 \\
\hline Headache* & 11.0 & 17.9 & 5.6 \\
\hline Other** & 35.4 & 44.6 & 28.2 \\
\hline
\end{tabular}

COVID-19-positive patients compared with negative patients with early recovery of smell function in a large proportion of patients. In our series, olfactory and gustatory impairments were respectively 14 times and 11 times more likely to be associated with
COVID-19 positivity. Overall, our findings suggest that loss of smell and loss of taste have a higher chance of identifying COVID-19-positive patients among those with influenza-like symptoms seen on an ambulatory basis. 


\begin{tabular}{|c|c|c|c|}
\hline \multicolumn{4}{|l|}{ Characterization of anosmia/hyposmia - \% } \\
\hline & Total $(n=127)$ & Positive $(n=56)$ & Negative $(n=71)$ \\
\hline \multicolumn{4}{|c|}{ Anosmia/hyposmia around the time of COVID-19 symptom onset* (5 days before or any time after) } \\
\hline Yes & 20.5 & 41.1 & 4.2 \\
\hline No & 64.6 & 41.1 & 83.1 \\
\hline Unable to recall & 13.4 & 16.1 & 11.3 \\
\hline Prefer not to respond & 0.8 & 0.0 & 1.4 \\
\hline Timing of anosmia/hyposmia & Total $(n=26)$ & Positive $(n=23)$ & Negative $(n=3)$ \\
\hline Before any other symptoms & 11.5 & 8.7 & 33.3 \\
\hline Early (after one or two other symptoms) & 42.3 & 43.5 & 33.3 \\
\hline Late (after other symptoms developed) & 42.3 & 43.5 & 33.3 \\
\hline Unknown (did not select) & 3.8 & 4.3 & 0.0 \\
\hline Current ability to smell & Total $(n=26)$ & Positive ( $n=23$ ) & Negative $(n=3)$ \\
\hline Normal & 50.0 & 47.8 & 66.7 \\
\hline Diminished & 23.1 & 26.1 & 0.0 \\
\hline Absent & 23.1 & 26.1 & 0.0 \\
\hline Unknown (did not select) & 3.9 & 0.0 & 33.3 \\
\hline \multicolumn{4}{|l|}{ Characterization of dysgeusia/ageusia - \% } \\
\hline & Total $(n=127)$ & Positive $(n=56)$ & Negative $(n=71)$ \\
\hline \multicolumn{4}{|c|}{ Dysgeusia/ageusia around the time of COVID-19 symptom onset* ( 5 days before or any time after) } \\
\hline Yes & 23.6 & 46.4 & 5.6 \\
\hline No & 69.3 & 51.8 & 83.1 \\
\hline Unable to recall & 6.3 & 1.8 & 9.9 \\
\hline Prefer not to respond & 0.8 & 0.0 & 1.4 \\
\hline Component of taste loss & Total $(n=30)$ & Positive $(n=26)$ & Negative $(n=4)$ \\
\hline Salty & 73.3 & 73.1 & 75.0 \\
\hline Sweet & 60.0 & 65.4 & 25.0 \\
\hline Sour & 56.7 & 57.7 & 50.0 \\
\hline Bitter & 60.0 & 61.5 & 50.0 \\
\hline Savoury & 76.7 & 80.8 & 50.0 \\
\hline
\end{tabular}

The reported prevalence of olfactory dysfunction varies significantly from $5.1 \%$ in a Chinese study to $85.6 \%$ in a European study. ${ }^{3-5,7-9}$ Our results are consistent with previous reports that showed a higher prevalence of self-reported smell or taste loss among COVID-19positive patients. Interestingly, in our study, although 31 out of $56(55.4 \%)$ of COVID-19-positive survey participants included anosmia or hyposmia as one of their overall symptoms, only 23 (41.1\%) patients maintained that they lost their sense of smell around the time of COVID-19 symptom onset (5 days prior or thereafter) with a $16 \%$ rate of "unable to recall." This discrepancy likely represents recall bias, which is common in crosssectional studies. Therefore, we believe that the $40 \%$ rate of smell loss around the time of COVID-19 symptom onset is a more accurate representation than the $55 \%$ overall rate.

\section{Comparison with previous studies}

Our rate of anosmia/hyposmia of $41.1 \%$ is lower than the rate of $60 \%-85 \%$ in recently published studies with comparable study designs or $51.5 \%$ found in another Canadian study. ${ }^{3,4,6,8}$ There may be several explanations for this difference. One consideration is the cultural difference in olfactory perception..$^{10-12}$ In fact, participants in other cultures may have different thresholds for their smell loss. Another possible explanation is the presence of mutant strains with varying pathogenicity, as evidenced from genomic studies in multiple nations. ${ }^{13-16}$ These different strains may cause varying degrees of chemosensory impairment with regional discrepancy, although this has not been scientifically proven. Therefore, it is important to collect and examine regional data to depict a representative landscape for our population. Lastly, we do note 


\begin{tabular}{|c|c|c|c|c|c|c|}
\hline & \multicolumn{2}{|c|}{ Univariable analysis } & \multicolumn{2}{|c|}{$\begin{array}{c}\text { Multivariable analysis with } \\
\text { anosmia/hyposmia }\end{array}$} & \multicolumn{2}{|c|}{$\begin{array}{c}\text { Multivariable analysis with } \\
\text { dysgeusia/ageusia }\end{array}$} \\
\hline & OR (95\% Cl) & $p$-value & OR $(95 \% \mathrm{Cl})$ & $p$-value & OR $(95 \% \mathrm{Cl})$ & $p$-value \\
\hline Age & $1.0(0.9-1.0)$ & 0.04 & & & & \\
\hline Gender & $1.2(0.6-2.4)$ & 0.7 & & & & \\
\hline Fever & $2.4(1.1-5.1)$ & 0.02 & $1.9(0.7-5.3)$ & 0.2 & $1.8(0.7-4.7)$ & 0.3 \\
\hline Cough & $2.7(1.3-5.6)$ & $<0.01$ & $1.8(0.7-5.2)$ & 0.3 & $2.2(0.9-5.8)$ & 0.1 \\
\hline Sore throat & $0.4(0.2-0.7)$ & $<0.01$ & $0.3(0.1-0.8)$ & 0.01 & $0.3(0.09-0.6)$ & $<0.01$ \\
\hline Rhinorrhea & $0.5(0.2-1.0)$ & 0.05 & & & & \\
\hline Nasal congestion & $1.1(0.6-2.3)$ & 0.7 & & & & \\
\hline Shortness of breath & $1.6(0.8-3.5)$ & 0.2 & & & & \\
\hline Fatigue & $0.4(0.1-1.3)$ & 0.2 & & & & \\
\hline Headache & $3.6(1.1-13.9)$ & 0.04 & $3.6(0.7-22.0)$ & 0.1 & $3.7(0.8-20.6)$ & 0.1 \\
\hline Abdominal pain or diarrhea & $2.2(1.0-5.0)$ & 0.04 & & & & \\
\hline Anosmia/hyposmia* & $19.7(6.1-88.7)$ & $<0.001$ & $14.4(4.0-70.5)$ & $<0.001$ & & \\
\hline Dysgeusia/ageusia* & $13.2(4.6-48.0)$ & $<0.001$ & & & $11.4(3.6-45.3)$ & $<0.001$ \\
\hline
\end{tabular}

that this difference could be a simple function of recall bias from cross-sectional surveys, as other studies do not report an "unable to recall" option, thus forcing patients to choose with an altered response rate of self-reported smell loss. Regardless, all published studies unanimously support the inclusion of smell and taste loss as important markers of COVID-19.

\section{Clinical and research implications}

A possible mechanism of olfactory dysfunction caused by SARS-CoV-2 has been demonstrated in experimental models with inoculation of coronaviruses. Coronaviruses can damage the olfactory neuroepithelium via apoptosis with a subsequent reduction of mature sensory neurons

\begin{tabular}{|c|c|c|c|}
\hline & Total $(n=108)$ & Anosmia/hyposmia ( $n=26$ ) & No anosmia/hyposmia $(n=82)$ \\
\hline Age - median (IQR) & $40.0(32.0-52.3)$ & $36.0(31.0-45.3)$ & $42.0(32.3-53.0)$ \\
\hline \multicolumn{4}{|l|}{ Smoking status - \% } \\
\hline Current & 7.4 & 3.9 & 8.5 \\
\hline Ex-smoker & 20.4 & 15.4 & 22.0 \\
\hline Never & 70.4 & 76.9 & 68.3 \\
\hline E-cigarettes & 1.9 & 3.9 & 1.2 \\
\hline \multicolumn{4}{|l|}{ Relevant comorbidities - \% } \\
\hline Chronic rhinosinusitis & 4.6 & 0.0 & 6.1 \\
\hline Recent severe URTI or flu & 11.1 & 15.4 & 9.8 \\
\hline Head trauma & 6.5 & 0.0 & 8.5 \\
\hline \multicolumn{4}{|l|}{ Relevant symptoms -\% } \\
\hline Rhinorrhea & 37.0 & 23.1 & 41.5 \\
\hline Nasal congestion & 40.7 & 46.2 & 39.0 \\
\hline \multicolumn{4}{|l|}{ COVID-19 status* - \% } \\
\hline Positive & 42.6 & 88.5 & 28.1 \\
\hline Negative & 57.4 & 11.5 & 72.0 \\
\hline
\end{tabular}


and disordering of olfactory epithelium. ${ }^{17-19}$ Another line of research suggests that the anosmia/hyposmia may result from infection of the support and perivascular cells in olfactory epithelium, via angiotensin-converting enzyme 2 receptors, which are identified as the receptors of SARS-CoV-2. ${ }^{20,21}$ Clinically, nasal inoculation of coronavirus resulted in olfactory impairment in healthy volunteers. $^{22}$ Dysgeusia/ageusia is generally regarded to be secondary to loss of smell, as it may be difficult to distinguish between flavor and taste. In a large study investigating taste disorders unrelated to COVID-19, many reported loss of taste without any objective gustatory loss but found to have olfactory deficits alone. ${ }^{23}$ While it is possible that loss of taste in COVID-19 may be a distinct mechanism, ${ }^{24}$ the rate of taste loss in our patients likely represents subtle olfactory deficits. In our series, $52 \%$ of the affected individuals reported a normal sense of smell at the time of survey with many recovering within 4 weeks. This finding is consistent with previous reports and suggests that the damage to the olfactory function by COVID-19 may not be permanent. ${ }^{4}$ However, this warrants further investigation with follow-up assessments of smell function. All in all, the presence of loss of smell and taste may be a distinguishable feature of COVID-19 from other viral URTIs.

Our results have strong implications in public health measures. A recent study demonstrated $56 \%$ of residents to be COVID-19 positive but "asymptomatic" in a skilled nursing facility. ${ }^{25}$ However, this study did not include the loss of smell or taste in the symptom assessment. In a previous study from Italy and our study, both demonstrate that a significant portion of the patients complained of decreased sense of smell or taste during the early phase of their disease. ${ }^{3}$ Hence, it would be essential to incorporate anosmia/hyposmia and dysgeusia/ageusia as part of future epidemiologic COVID-19 surveillance studies and tailor the screening criteria for COVID-19 accordingly. Furthermore, these symptoms can be easily assessed in the context of telemedicine and virtual care. This can, in turn, facilitate improved patient counselling for the screening of COVID-19 and help curb the spread of COVID-19 in the vulnerable population, including those in long-term care facilities and remote communities where testing may be limited. Currently, while several provincial bodies have implemented loss of smell/taste as screening symptoms, the self-assessment tool from the Canadian Federal Government still does not incorporate these symptoms. With our Ontario regional data demonstrating a high probability of COVID-19 positivity with loss of smell/ taste, there is now evidence to expand public awareness of this association and ultimately improve our public health response to this pandemic.

\section{Limitations}

Interpretation of our study results is limited by recall bias, as there is a significant time lapse between diagnosis and survey administration. The recent media spotlight of COVID-19 and anosmia might have resulted in elevated rates of self-reported anosmia. We tried to mitigate this issue by asking similar, but differently worded, questions for internal validity. Future studies could be administered at the time of swab to avoid this bias. We also acknowledge non-response bias. While the study results are limited by the nature of a single-institutional-based study, we collected data over a longer period of time than previous reports to improve our sampling. ${ }^{3,4}$ Furthermore, the testing criteria focused on frontline workers from at-risk settings, such as healthcare or long-term care, residents in group homes, and returning travelers. This may reduce external validity. Lastly, with ethical concerns of transmission to our research team and preservation of personal protective equipment, we are presently unable to objectively measure smell in symptomatic patients during the point of assessment.

We acknowledge the need for large-scale epidemiologic studies to further investigate this association between smell and taste loss and COVID-19 positivity. Our research group currently has ongoing projects to investigate a larger regional population and to incorporate selfreported objective measures of smell using common household items. Despite the limitations, the baseline characteristics between positive and negative groups were comparable, and our data emphasize the importance of considering smell and taste as screening symptoms.

\section{CONCLUSION}

Our results demonstrate that there is a higher rate of selfreported anosmia/hyposmia and concurrent dysgeusia/ ageusia among COVID-19 patients in a regional sample of Canadians in Toronto, Ontario. Our study offers strong support for clinicians to use loss of smell and loss of taste as key symptoms for the clinical diagnosis of COVID-19, especially in settings with limited testing. 
Future population-based studies are needed to further investigate this association.

Acknowledgements: The authors sincerely thank Cheryl Pedersen and the Survey Research Unit at St. Michael's Hospital for survey administration and study support and The Dunin Foundation for research support. We would like to acknowledge Drs. Jonathan Overdevest and Stuart Firestein for the initial survey design. We would like to thank Dr. Jennifer Anderson for her support of this study.

\section{Author contributions:}

Study concept and design: D. Lee, J. Lockwood, P. Das, R. Wang, E. Grinspun, J. Lee

Acquisition of data: D. Lee, J. Lockwood, P. Das, J. Lee Analysis and interpretation of data: D. Lee, R. Wang, J. Lee Initial draft of the manuscript: D. Lee

Critical revision of the manuscript for important intellectual content: D. Lee, J. Lockwood, P. Das, E. Grinspun, R. Wang, J. Lee Administrative, technical or material support: $\mathrm{D}$. Lee, J. Lee Study supervision: J. Lee

All authors have given their agreement for this study.

Financial support: This study was funded by The Dunin Foundation.

Competing interests: JML has received honoraria from Baxter Corporation. All other authors have declared no potential conflicts of interest.

\section{REFERENCES}

1. Gandhi RT, Lynch JB, del Rio C. Mild or moderate COVID-19. N Engl 7 Med 2020;epub, doi:10.1056/ NEJMcp2009249.

2. Bai Y, Yao L, Wei T, et al. Presumed asymptomatic carrier transmission of COVID-19. FAMA 2020;323(14):1406-7.

3. Spinato G, Fabbris C, Polesel J, et al. Alterations in smell or taste in mildly symptomatic outpatients with SARS-CoV-2 infection. FAMA 2020;epub, doi:10.1001/jama.2020.6771.

4. Yan CH, Faraji F, Prajapati DP, Boone CE, DeConde AS. Association of chemosensory dysfunction and COVID-19 in patients presenting with influenza-like symptoms. Int Forum Allergy Rbinol 2020;epub, doi:10.1002/alr.22579.

5. Moein ST, Hashemian SM, Mansourafshar B, et al. Smell dysfunction: a biomarker for COVID-19. Int Forum Allergy Rbinol 2020;epub, doi:10.1002/alr.22587.

6. Carignan A, Valiquette L, Grenier C, et al. Anosmia and dysgeusia associated with SARS-CoV-2 infection: an age-matched case-control study. CMA7 2020;epub, doi:10.1503/cmaj. 200869.

7. Vroegop VA, Eeckles AS, Van Rompaey, V, et al. COVID-19 and olfactory dysfunction - an ENT perspective to the current COVID-19 pandemic. B-ENT 2020;16:81-85. doi: 10.5152/B-ENT.2020.20127.

8. Lechien JR, Chiesa-Estomba CM, De Siati DR, et al. Olfactory and gustatory dysfunctions as a clinical presentation of mild-to-moderate forms of the coronavirus disease (COVID-19): a multicenter European study. Eur Arch Otorbinolaryngol 2020;epub, doi:10.1007/s00405-020-05965-1.
9. Vaira LA, Salzano G, Deiana G, De Riu G. Anosmia and ageusia: common findings in COVID-19 patients. Laryngoscope 2020;epub, doi:10.1002/lary.28692.

10. Candau J. The olfactory experience: constants and cultural variables. Water Sci Technol 2004;49(9):11-7.

11. Ferdenzi C, Joussain P, Digard B, et al. Individual differences in verbal and non-verbal affective responses to smells: influence of odor label across cultures. Chem Senses 2017;42(1):37-46.

12. Ferdenzi C, Roberts SC, Schirmer A, et al. Variability of affective responses to odors: culture, gender, and olfactory knowledge. Chem Senses 2013;38(2):175-86.

13. Gudbjartsson DF, Helgason A, Jonsson H, et al. Spread of SARS-CoV-2 in the Icelandic population. $N$ Engl $7 \mathrm{Med}$ 2020; epub, doi:10.1056/NEJMoa2006100.

14. Su Y, Anderson D, Young B, et al. Discovery of a 382-nt deletion during the early evolution of SARS-CoV-2. bioRxiv 2020;epub, doi:10.1101/2020.03.11.987222.

15. Xi J, Xu K, Jiang P, et al. Virus strain of a mild COVID-19 patient in Hangzhou representing a new trend in SARS$\mathrm{CoV}-2$ evolution related to Furin cleavage site. medRxiv 2020;epub, doi:10.1101/2020.03.10.20033944.

16. Yao H, Lu X, Chen Q, et al. Patient-derived mutations impact pathogenicity of SARS-CoV-2. medRxiv 2020;epub, doi: 10.1101/2020.04.14.20060160.

17. Mori I, Goshima F, Imai Y, et al. Olfactory receptor neurons prevent dissemination of neurovirulent influenza A virus into the brain by undergoing virus-induced apoptosis. $7 \mathrm{Gen}$ Virol 2002;83(9):2109-16.

18. Mori I, Nishiyama Y, Yokochi T, Kimura Y. Virus-induced neuronal apoptosis as pathological and protective responses of the host. Rev Med Virol 2004;14(4):209-16.

19. Schwob JE, Saha S, Youngentob SL, Jubelt B. Intranasal inoculation with the olfactory bulb line variant of mouse hepatitis virus causes extensive destruction of the olfactory bulb and accelerated turnover of neurons in the olfactory epithelium of mice. Chem Senses 2001;26(8):937-52.

20. Brann D, Tsukahara T, Weinreb C, Logan DW, Datta SR. Non-neural expression of SARS-CoV-2 entry genes in the olfactory epithelium suggests mechanisms underlying anosmia in COVID-19 patients. bioRxiv 2020;epub, doi:10.1101/ 2020.03.25.009084.

21. Zhou P, Yang X-L, Wang X-G, et al. A pneumonia outbreak associated with a new coronavirus of probable bat origin. Nature 2020;579(7798):270-3.

22. Åkerlund A, Bende M, Murphy C. Olfactory threshold and nasal mucosal changes in experimentally induced common cold. Acta Otolaryngol 1995;115(1):88-92.

23. Deems DA, Doty RL, Settle RG, et al. Smell and taste disorders, a study of 750 patients from the University of Pennsylvania Smell and Taste Center. Arch Otolaryngol Head Neck Surg 1991;117(5):519-28.

24. Vaira LA, Salzano G, Fois AG, Piombino P, De Riu G. Potential pathogenesis of ageusia and anosmia in COVID-19 patients. Int Forum Allergy Rhinol 2020;epub, doi:10.1002/ alr.22593.

25. Arons MM, Hatfield KM, Reddy SC, et al. Presymptomatic SARS-CoV-2 infections and transmission in a skilled nursing facility. NEngl7 Med 2020;epub, doi:10.1056/NEJMoa2008457. 